\title{
Über die Gleichartigkeit von Kartoffelstärken verschiedener Herkunft
}

\author{
JAKOB BLOM und BIRGITSCHWARZ
}

Laboratorium der Tuborg-Brauereien, Kopenhagen, Dänemark

\begin{abstract}
Qtärkekleister wird bei der Einwirkung von $\alpha$-Amylasen dünnflüssig. Diese Reaktion benützt man zur Bestimmung der Aktivität von $\alpha$-Amylasepräparaten, zum Beispiel in der von Blom und Bak ${ }^{1}$ ausgearbeiteten Methode. Das gleiche $\alpha$-Amylasedauerpräparat gab nach dieser Methode untersucht dieselbe Enzymaktivität bei Verwendung 14 verschiedener Proben von prima Kartoffelstärke. Der mittlere Fehler betrug $\pm 1 \%$. Die Stärken stammten aus 6 verschiedenen europäischen Ländern und waren aus Kartof-
\end{abstract} feln der Ernten 1929 bis 1937 hergestellt.

Stärkekleister wird bei der Einwirkung von $\alpha$ - und $\beta$-Amylasen verzuckert. Diese Reaktion benützt man zur Bestimmung der diastatischen Kraft. Hierzu verwendet man Lösungen von löslicher Stärke. Verschiedene Handelspräparate löslicher Stärke geben mit demselben Amylasepräparat verschiedene Werte der Aktivität. Nach dem Kriege war es in Dänemark unmöglich, lösliche Stärke zu beziehen. Anstatt lösliche Stärke selbst herzustellen, welche a priori mit dem soeben genannten Mangel behaftet ist, haben wir mit Erfolg einen anderen Weg eingeschlagen. Wir haben eine Methode zur Bestimmung der diastatischen Kraft unter Verwendung unbehandelter Kartoffelstärke ausgearbeitet ${ }^{2}$. Das gleiche Malzamylasedauerpräparat gab, nach dieser Methode untersucht, dieselbe Enzymaktivität bei Verwendung 18 verschiede- . ner Proben von prima Kartoffelstärke. Die Stärken stammten aus 10 verschiedenen europäischen Ländern und waren aus Kartoffeln der Ernten 1931 bis 1948 hergestellt. Der mittlere Fehler betrug $\pm 1 \%$ mit unbehandelter Kartoffelstärke, mit löslicher Stärke dagegen $\pm 5 \%$.

In der vorhergehenden Arbeit ${ }^{3}$ wurde gezeigt, dass beim Abbau von Kleister aus unbehandelter Kartoffelstärke durch $\alpha$-Amylase aus Malz und aus Bacillus subtilis unvergärbare reduzierende Dextrine in einer maximalen 
Tabelle 1. Abbau von Kartoffelstärke verschiedener Provenienz durch $\alpha$-Subtilisamylase. Differenzierung der Abbauprodukte durch $\beta$-Gerstenamylase und durch Gärung mit S. carlbergensis. (1950) Kleister $2,00 \%, \quad p H=5,0, t=40,0^{\circ}$

Reduktionsvermögen, ber. als Maltose, \% TM, nach Blom und Rosted.

\begin{tabular}{|c|c|c|c|c|c|c|c|}
\hline \multirow{2}{*}{$\begin{array}{l}\text { Bezeich- } \\
\text { nung }\end{array}$} & \multirow{2}{*}{ Provenienz } & \multirow{2}{*}{ Ernte } & \multicolumn{2}{|c|}{$\alpha$-Amylase } & \multicolumn{2}{|c|}{$\begin{array}{c}\beta \text {-Amylase nach } \\
\alpha \text {-Amylase und Gärung }\end{array}$} & \multirow{2}{*}{$\begin{array}{c}\text { Durch } \\
\beta \text {-Amylase } \\
\text { spaltbare } \\
\alpha \text {-Dextrine }\end{array}$} \\
\hline & & & $\begin{array}{c}\text { Vor } \\
\text { Gärung }\end{array}$ & $\begin{array}{l}\text { Nach } \\
\text { Gärung }\end{array}$ & $\begin{array}{l}\text { Vor } \\
\text { Gärung }\end{array}$ & $\begin{array}{l}\text { Nach } \\
\text { Gärung }\end{array}$ & \\
\hline $\mathbf{S}$ & Norwegen & 1947 & 37,7 & 20,6 & 71,7 & 2,6 & 18,0 \\
\hline $\mathbf{Y}$ & England & 1947 & 37,7 & 21,0 & 69,1 & 2,8 & 18,2 \\
\hline $\mathbf{Q}$ & Frankreich & 1947 & 39,1 & 21,1 & 69,9 & 2,8 & 18,3 \\
\hline DK & Dänemark & 1948 & 39,2 & 22,0 & 69,9 & 4,1 & 17,9 \\
\hline $\mathrm{E}$ & Dänemark & 1937 & 39,8 & 21,0 & 69,1 & 3,5 & 17,5 \\
\hline $\bar{Z}$ & Tschechoslowakei & 1947 & 40,0 & 21,5 & 71,6 & 2,6 & 18,9 \\
\hline $\mathbf{A}$ & Deutschland & 1931 & 40,5 & 21,8 & 69,1 & 3,3 & 18,5 \\
\hline $\mathrm{B}$ & Holland & 1933 & 40,6 & 21,7 & 69,6 & 3,4 & 18,3 \\
\hline $\mathrm{C}$ & Holland & 1936 & 40,8 & 21,6 & 70,4 & 3,0 & 18,6 \\
\hline Ho & Holland & 1947 & 41,7 & 22,0 & 72,6 & 3,8 & 18,2 \\
\hline $\mathrm{C}$ & Holland & 1936 & 41,9 & 21,7 & 69,1 & 3,1 & 18,6 \\
\hline I & Schweden & 1937 & 42,8 & 21,4 & 69,2 & 2,6 & 18,8 \\
\hline $\mathbf{A}$ & Deutschland & 1931 & 46,7 & 22,1 & 69,2 & 3,5 & 18,6 \\
\hline \multicolumn{3}{|c|}{ Mittel } & & 21,5 & & 3,2 & 18,3 \\
\hline
\end{tabular}

Anzahl von $21 \%$ TM* gebildet werden. Smits van Waesberghe ${ }^{4} \mathrm{kam}$ zu demselben Ergebnis. Bei einer darauf folgenden Behandlung mit $\beta$-Gerstenamylase konnten wir zeigen, dass 18 von den 21 Dextrinen in vergärbare Spaltprodukte umgewandelt werden.

Eine Reihe von Proben von prima Kartoffelstärke aus unserer Sammlung wurde wie in der vorhergehenden Arbeit untersucht. Der Stärkekleister wird durch $\alpha$-Amylase aus B.subtilis zu etwa $40 \%$ TM abgebaut und das Reaktionsgemisch darauf mit Bierhefe vergoren. Der Abbau wird nunmehr mit $\beta$-Amylase aus Gerste fortgesetzt und das Reaktionsgemisch wiederum mit Bierhefe vergoren. Das Reduktionsvermögen wird nach Einwirkung jeden Enzyms vor und nach der Gärung nach der Kupferkarbonatmethode von Blom und Rosted ${ }^{5}$ bestimmt.

Im ganzen wurden 13 Proben von Kartoffelstärke untersucht. Die Stärken stammten aus 8 verschiedenen Ländern und waren aus Kartoffeln der Ernten 1931 bis 1948 hergestellt. In allen Fällen gab die Untersuchung dasselbe Resultat. $a$-Subtilisamylase bildet unvergärbare Dextrine in einer Anzahl von 21,5\%

* $\% \mathrm{TM}=\%$ der theoretisch möglichen Menge Maltose, die gebildet werden würde, falls Stärke sich vollständig zu Maltose abbauen liesse. 
TM. 18,3 von diesen werden durch $\beta$-Amylase zu vergärbaren Spaltprodukten abgebaut.

Aus der ersten Versuchsreihe über die Verflüssigung, aus der zweiten über die Verzuckerung und aus der dritten über die maximale Anzahl der Dextrine darf man den Schluss ziehen, dass weder Klima, Boden oder Sorte einen Einfluss auf die Konstitution der Kartoffelstärke haben.

\section{Z USAMMENFASSUNG}

Beim Abbau von Kartoffelstärkekleister durch $\alpha$-Amylase aus $B$. subtilis werden durch Bierhefe vergärbare und unvergärbare Spaltstücke gebildet. Die Anzahl der unvergärbaren Spaltstücke, Dextrine, erreicht ein Maximum. Die Dextrine lassen sich zum grössten Teil durch $\beta$-Gerstenamylase zu vergärbaren Spaltstücken abbauen.

Es sind 13 Proben von Kartoffelstärke aus 8 verschiedenen Ländern der Ernten 1931 bis 1948 untersucht, in allen Fällen mit demselben Resultat. $\alpha$-Subtilisamylase bildet unvergärbare Dextrine in einer Anzahl von 21,5 \% TM. 18,3 von diesen werden durch $\beta$-Amylase zu vergärbaren Spaltprodukten abgebaut.

Aus früheren Versuchen über die Verflüssigung (14 Proben) und über die Verzuckerung (18 Proben) von Stärkekleister durch Amylasen und aus obigen Versuchen lässt sich schliessen, dass weder Klima, Boden oder Sorte einen Einfluss auf die Konstitution der Kartoffelstärke haben.

\section{IITERATUR}

1. Blom, J. und Bak, A. Z. physiol. Chem. 256 (1938) 197.

2. Blom, J. und Schwarz, B. 1. Int. Congr. of Biochem. Cambridge (1949) 562; J. Inst. Brewing (1950) 58.

3. Blom, J., Schmith, T. und Schwarz, B. Acta Chem. Scand. 6 (1952) 591.

4. Smits van Waesberghe, F. A. M. J. Onderzoekingen over Microben-Amylasen. (1941). Delft.

5. Blom, J. und Rosted, C. O. Acta Chem. Scand. 1 (1947) 32.

Eingegangen am 14. März 1952. 